\title{
IMPROVING THE INHALATION TECHNIQUE IN PATIENTS WITH OBSTRUCTIVE PULMONARY DISEASES
}

\author{
Zlatina I. Ivanova, \\ Nikolay H. Kyuchukov, \\ Iliya I. Krachunov, \\ Nikolay A. Yanev, \\ Pavlina Ts. Glogovska, \\ Plamen S. Pavlov, \\ Tsanya P. Popova ${ }^{1}$, \\ Vasil V. Todorov', \\ Yavor Y. Ivanov \\ Department of Cardiology, \\ Pulmonology and Endocrinology, \\ Medical University - Pleven, \\ Bulgaria \\ ${ }^{1}$ Department of Propaedeutics of \\ Internal Diseases, \\ Medical University - Pleven, \\ Bulgaria \\ ${ }^{2}$ Department of Nephrology, \\ Haematology and Gastroenterology, \\ Medical University - Pleven, \\ Bulgaria
}

\section{Corresponding Author:}

Zlatina I. Ivanova

Clinic of Pneumonology and Phthysiatry, University Hospital "Dr. Georgi Stranski” 91, Gen. Vl. Vazov Blvd.

Pleven, 5800

Bulgaria

e-mail: zlati.dikova@gmail.com

Received: June 14, 2016

Revision received: October 10, 2016

Accepted: December 20, 2016

\section{Summary}

The study aimed to assess the inhalation technique of patients with bronchial asthma/chronic obstructive pulmonary disease (COPD) via an objective method and to evaluate the effect of training in patients with incorrect technique. The inhalation technique of 120 patients with obstructive pulmonary disease was tested. The patients were divided into two groups: using metered dose inhalers (MDI) - 34 patients $(28 \%)$ and dry powered inhalers (DPI) -86 patients $(72 \%)$. The most frequent mistakes in the MDI group were short duration of the inhalation $(55.88 \%)$ and bad synchronization between activating the canister and the inhalation (29.41\%). For the DPI group, the inhalation was not forceful enough $(48.84 \%)$ and the short duration of the inhalation $(12.79 \%)$. Patients claiming to have good inhalation technique accounted for $97 \%$ of those in the MDI group, and $96.5 \%$ of those in the DPI group. There were two patients $(5.88 \%)$ with correct inhalation technique in the MDI group at their first attempt, and 31 patients $(36.05 \%)$ in the DPI group. We found that in the MDI group there was a significant reduction in the number of mistakes $(\mathrm{p}<0.001)$. In the DPI group, such correlation was not found but during visit 2 there were no patients with more than 1 mistake. Correcting poor inhalation technique led to reduction of the number of mistakes during inhalation.

Key words: inhaler, obstructive diseases, drug misuse

\section{Introduction}

The term "inhaler" was first used in 1778 for a device - a metal mug with a tap for opium vapour inhalation for coughing. In 1858, Sales-Girons invented the first pressurized inhaler and in 1864, Newton constructed the first dry powdered inhaler [1]. Today, inhalation therapy remains one of the most important parts in the treatment of obstructive pulmonary diseases. There are two main types of inhalers - dry powder inhalers (DPIs), which are preferred in Europe [2] and pressurised metered dose inhalers (pMDIs).

Inhalation is thought to be the optimal way to treat obstructive pulmonary diseases as it provides high drug concentrations in the lungs with minimal systemic side effects [3]. There are a number of 
reports in the literature about patients unable to use their inhaler devices properly. This may lead to poor treatment results and increased cost extra emergency visits, hospitalizations, extra therapy [2]. On the other hand, good inhalation technique leads to good control of the obstructive disease, rarer use of reliever medications, higher lung volumes and better quality of life [2].

It has been found that patients, who do not use spacers, have worse inhaler technique [2]. Sex and age also have an effect on inhalation skills [4]. Female patients have more difficulties using MDI devices [4, 5]. Older patients, those with lower education and people, who had not received enough instructions about their inhaler use, have been reported to have worse inhalation skills $[4,6]$.

There are similar observations in Bulgaria, even among children with bronchial asthma. Worse asthma control could be due to difficulties, which children and their parents encounter. Reliable and easy-to-use devices could contribute for better control of obstructive disease [7].

Leading guidelines for asthma treatment [8] place emphasis on the importance of achieving and keeping the disease symptoms under control. Asthma control includes clinical control (minimal symptoms, optimal quality of life) and preventing the risk of future exacerbations and decrease of lung volumes [8]. For clinical followup of asthma control, a score system could be used, which expresses the patient's health for a period, not only for the present moment, e.g. questions from Asthma Control Test (ACT) concerning the health condition for the previous 4 weeks [9]. In patients with chronic obstructive pulmonary disease (COPD), the impact of the symptoms (cough, sputum, fatigue, dyspnea) could be assessed via the COPD Assessment Test (CAT) [10].

Education of patients with obstructive pulmonary diseases is part of their treatment. In patients with COPD, the educational program depends on the severity of the disease but usually it includes general information about COPD, risk factors and complications of the disease, inhalers for treatment and home oxygen therapy, and early recognition of exacerbation symptoms [11].

To our knowledge, up to now there are no publications in Bulgaria concerning the correct inhaler technique, tested with an objective method. There are devices, constructed for testing the inhaler technique objectively. One such device is Vitalograph Aerosol Inhalation
Monitor (AIM), with which the most important steps during inhalation could be easily assessed: synchronization between activating the inhaler and inspiration, optimal airflow (strength and duration), and holding the breath for at least 5 seconds [2].

The study aimed to assess the inhalation technique of patients with obstructive pulmonary disease (bronchial asthma and COPD) via an objective method and to evaluate the effect of the education of patients with incorrect inhalation technique.

\section{Patients and Methods}

The study was approved by the Ethical Committee at Medical University Pleven. Over a one-year period (April 2015 - May 2016), 120 patients with obstructive pulmonary disease (COPD/ bronchial asthma), treated in the Clinic of Pneumonology and Phthysiatry, University Hospital Pleven, were included in the study. During visit 1 , short questionnaires - ACT (for patients with bronchial asthma) and CAT for patients with COPD respectively, were filled on discharge after patients had signed an informed consent. The maximum score for ACT was 25 points, with assessment scale as follows: 25 points - good asthma control, 20-24 points satisfactory asthma control and below 20 points unsatisfactory control. The maximum score for CAT was 40 points. The higher the score, the more symptomatic the patient was.

The inhalation technique was evaluated via an objective method - AIM (Vitalograph $®$ ). The Inhalation monitor consisted of a control device, a silicone connecting tube and a single-use disposable inhaler simulator - DPI or MDI with placebo canisters (Figure 1).

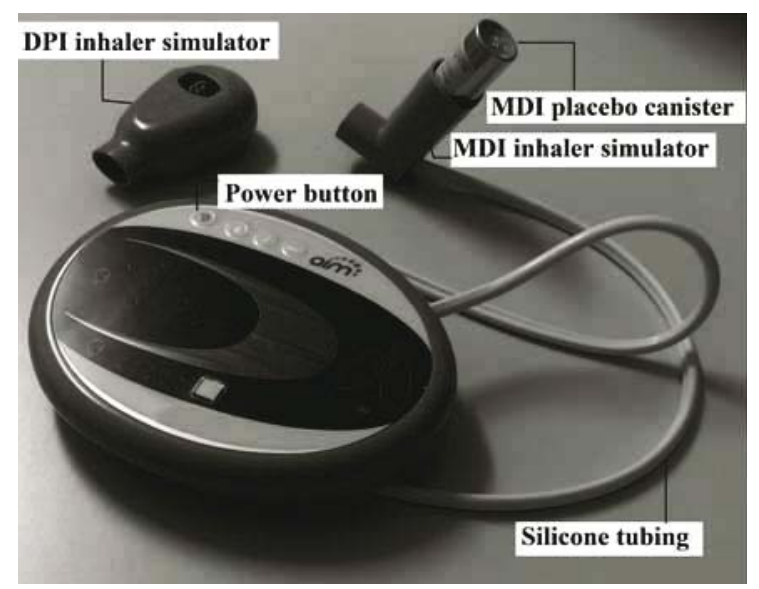

Figure 1. Main components of the AIM devices 
The inhaler simulator we used was matched to the type of the patients' inhalation devices. The inhalation technique assessment had several components: keeping the lips tightly around the mouthpiece, good synchronization between activating the placebo canister and inhalation (only for MDI devices), optimal airflow during inhalation (strength and duration of inhalation) and holding the breath after inhalation for at least 5 seconds. Patients made up to two attempts to test their technique. Those demonstrating correct inhalation techniques received a certificate for participation in the study. The patients who had incorrect inhalation technique were further educated, their mistakes were discussed with the investigator and a second visit (visit 2) took place. The test results were divided in the following groups: 1) synchronization between activation of the canister and the inhalation; 2) not inhaling forcefully enough; 3 ) low duration of the inhalation; 4) not holding the breath after inhalation for at least 5 seconds; 5) not keeping the lips tightly around the mouthpiece; 6) good inhalation technique; 7) other mistakes. During visit 2 , the patients had to demonstrate their newly acquired inhalation skills. The patients, in whom incorrect inhalation technique was still observed, were tested with the alternative inhaler stimulator. A recommendation note was written to the pulmonologist who followed up the patient on an outpatient basis to consider changing the type of the controller inhalator.

Data was entered into an Excel table and was processed statistically with Statgraphics Plus. Mean and median (ME) [standard deviation (SD)] were used to describe patients' characteristics and proportions. Other statistical tests $-\chi^{2}$ test and Kruskal-Wallis test $(\mathrm{KW})$ were used where appropriate.

\section{Results}

In this study, the inhalation technique of 120 patients with obstructive pulmonary diseases was tested. The patients were divided in two groups, according to the type of their controller device: with MDI - 34 (28\%) patients, and with DPI - 86 $(72 \%)$ patients. In the MDI group, the average age was 57.5 years (age range 26-82). In the DPI group, the average age was 63 years (age range 26-83). The patients in the MDI group were significantly younger than the patients in the DPI group $(\mathrm{KW}=5.584 ; \mathrm{p}=0.018)$. The patients with bronchial asthma were 54 ( $45 \%$ of all patients): 22 of them (40.74\%) used MDI devices and 32 (59.26\%) - DPI devices (Table 1). The patients with COPD were 66 (55\%). Those using MDI

Table 1. Some characteristics of the patients from the MDI and DPI groups

\begin{tabular}{|c|c|c|c|}
\hline & $\begin{array}{l}\text { MDI } \S \text { group } \\
n_{1}=34(28 \%) \text { patients }\end{array}$ & $\begin{array}{l}\text { DPI* group } \\
n_{2}=86(72 \%) \text { patients }\end{array}$ & \\
\hline $\begin{array}{l}\text { Age yrs } \dagger \text { ME } \ddagger \\
(\min -\max )\end{array}$ & $\begin{array}{l}57.5 \mathrm{yrs} \\
(26-82)\end{array}$ & $\begin{array}{l}63 \mathrm{yrs} \\
(26-83)\end{array}$ & $\mathrm{p}=0.018$ \\
\hline $\begin{array}{l}\text { Bronchial asthma } \\
\text { (54 patients) }\end{array}$ & $22(40.74 \%)$ & $32(59.26 \%)$ & \multirow{2}{*}{$\mathrm{p}=0.012$} \\
\hline $\begin{array}{l}\text { COPD } \neq \\
\text { (66 patients) }\end{array}$ & $12(18.18 \%)$ & $54(81.82 \%)$ & \\
\hline \multicolumn{4}{|l|}{ Using spacer? } \\
\hline Yes & $6(17.7 \%)$ & & \\
\hline No & $28(82.3 \%)$ & & \\
\hline \multicolumn{4}{|c|}{$\begin{array}{l}\text { Good inhalation skills } \\
\text { according to the patients? }\end{array}$} \\
\hline Yes & $33(97 \%)$ & $83(96.5 \%)$ & \\
\hline No & $1(3 \%)$ & $3(3.5 \%)$ & \\
\hline
\end{tabular}

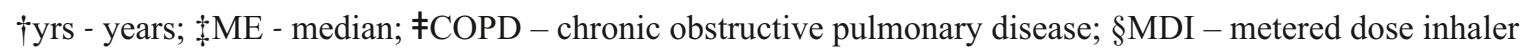
*DPI - dry powder inhaler 
devices were $12(18.18 \%)$, and $54(81.82 \%)$ used DPI. Patients with COPD, using DPI devices were significantly more than the patients with COPD and MDI devices $\left(\chi^{2}=6.37 ; p=0.012\right)$. It was found that in the MDI group, 6 patients $(17.7 \%)$ had a spacer but only 2 of them used it regularly.

The patients claiming to have good inhalation technique accounted for $97 \%$ of those in the MDI group and $96.5 \%$ for the patients in the DPI group (Table 1).

The most frequent mistakes in the MDI group were short duration of the inhalation (55.88\%) and poor synchronization between the canister activation and the inhalation itself (29.41\%, Figure 2A). The most common mistakes in the DPI group were that the inhalation was not forceful enough $(48.84 \%)$ and it was of short duration (12.79\%, Figure 2B). In the MDI group, only $2(5.88 \%)$ of the patients demonstrated correct inhalation technique during their first attempt. In the DPI group, the patients with correct inhalation at their first attempt were 31 $(36.05 \%)$. There were some patients, who made more than one mistake during inhalation. At visit 1, patients with MDI made more mistakes (on the average, 1 patient made 2 mistakes) during inhalation than patients with DPI device (on the average 1 patient made 1 mistake) (KW=11.892; $\mathrm{p}<0.001)$. Patients, who demonstrated a correct inhalation technique from their first or second attempt (49 patients with DPI and 8 patients with MDI), were considered as ones with correct inhalation technique and received a certificate for participation and good inhalation skills. For the rest of the patients, a second visit for retesting the

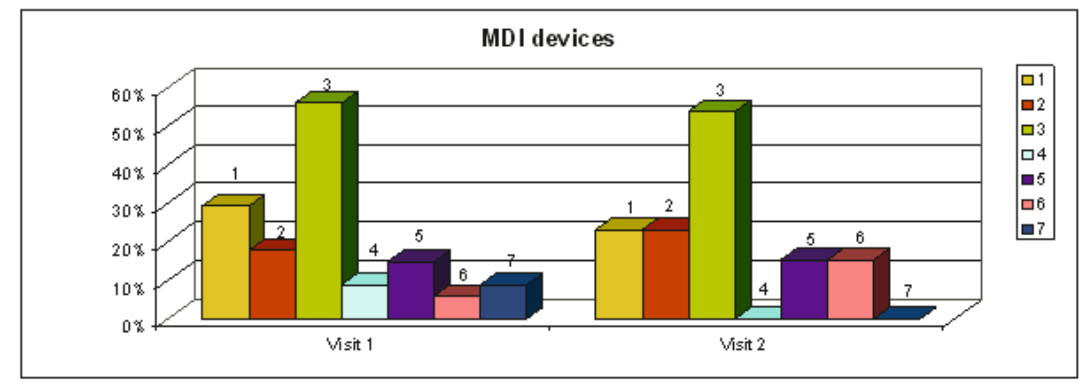

Figure 2A. Most common mistakes made with MDI devices on visit 1 and visit 2 . At visit 1 , the most frequent mistakes in the MDI group were short duration of the inhalation (55.88\%) and poor synchronization between canister activation and the inhalation (29.41\%). At visit 2, most common mistake for MDI devices was the short duration of the inhalation

1) poor synchronization between the placebo canister activation and the inhalation; 2) not inhaling enough forcefully; 3) low duration of the inhalation; 4) not holding the breath after inhalation for at least 5 seconds; 5) not keeping the lips tightly around the mouthpiece; 6) good inhalation technique; 7) other mistakes

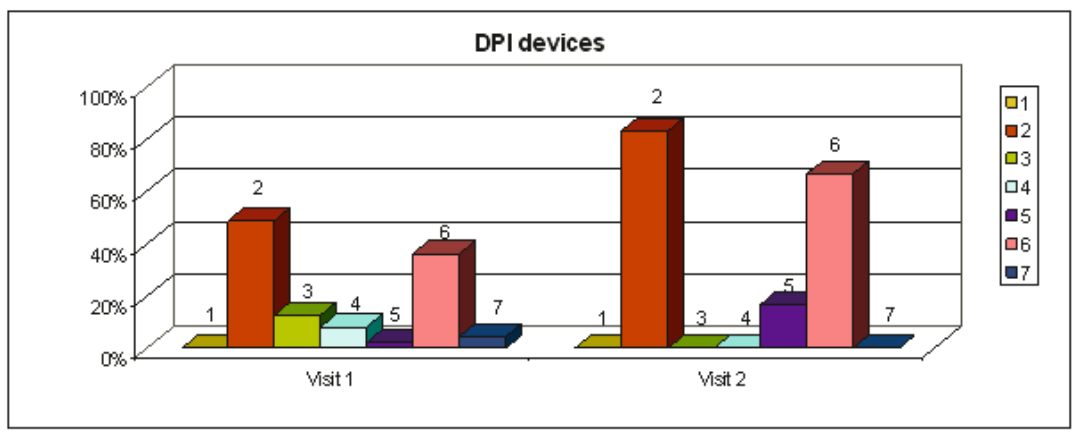

Figure 2B. Most common mistakes made with DPI devices on visit 1 and visit 2. At visit 1 the most common mistakes in the DPI group were that the inhalation was not forceful enough $(48.84 \%)$ and the short duration of the inhalation $(12.79 \%$ ). During visit 2 the most frequent mistake for the DPI devices was that the inhalation was not forceful enough $(20 \%)$

1) poor synchronization between activating the placebo canister and the inhalation; 2) not inhale enough forcefully; 3) low duration of the inhalation; 4) holding the breath after inhalation for at least 5 seconds; 5) not keeping close the lips tightly around the mouthpiece; 6) good inhalation technique; 7) other mistakes There are no patients with DPI and bad coordination between activating the canister and the inhalation, because this option is not available in DPI devices 
inhalation technique was appointed.

At Visit 2, 38 patients did not turn up, and 25 ( $20.83 \%$ of all patients) attended the visit. At Visit 2, 6 patients demonstrated correct inhalation technique at the first attempt. At this visit, the most common mistake for MDI devices was short duration of the inhalation, observed in $7(28 \%)$ patients and for DPI devices - the inhalation was not forceful enough in $5(20 \%)$ cases (Figures $2 \mathrm{~A}$ and $2 \mathrm{~B}$ ).

In 7 patients ( 3 with MDI and 4 with DPI), good inhalation technique could not be demonstrated at all. A recommendation note for changing the type of inhaler device was written after the patients had been trained again. There was no difference between the two groups as regards the average number of mistakes at Visit 2: for both groups, there was one mistake per patient on the average $(\mathrm{KW}=0.333 ; \mathrm{p}=0.564)$.

When comparing the number of mistakes in the MDI group at Visit $1(\mathrm{ME}$ [SD] $=2[0.87])$ and for Visit $2(\mathrm{ME}[\mathrm{SD}]=1[0.38])$ it was found that patients made significantly more mistakes during visit $1(\mathrm{KW}=7.848 ; \mathrm{p}=0.005)$. For the DPI group during Visit 1, 44 (80\%) patients made 1 mistake; $11(18.18 \%)$ - 2 mistakes; and only $1(1.82 \%)$ made 3 mistakes. At visit 2, all the 6 people made 1 mistake (Figure 3). Even though there was no significant correlation between the number of mistakes at visit 1 and 2 in the DPI group (Visit 1 $\mathrm{ME}[\mathrm{SD}]=1$ [0.46]; Visit $2 \mathrm{ME}[\mathrm{SD}]=1[0.41]$; $\mathrm{KW}=0.044 ; \mathrm{p}=0.833$ ) we might suggest that patients with more than 1 mistake were not seen at visit 2 .
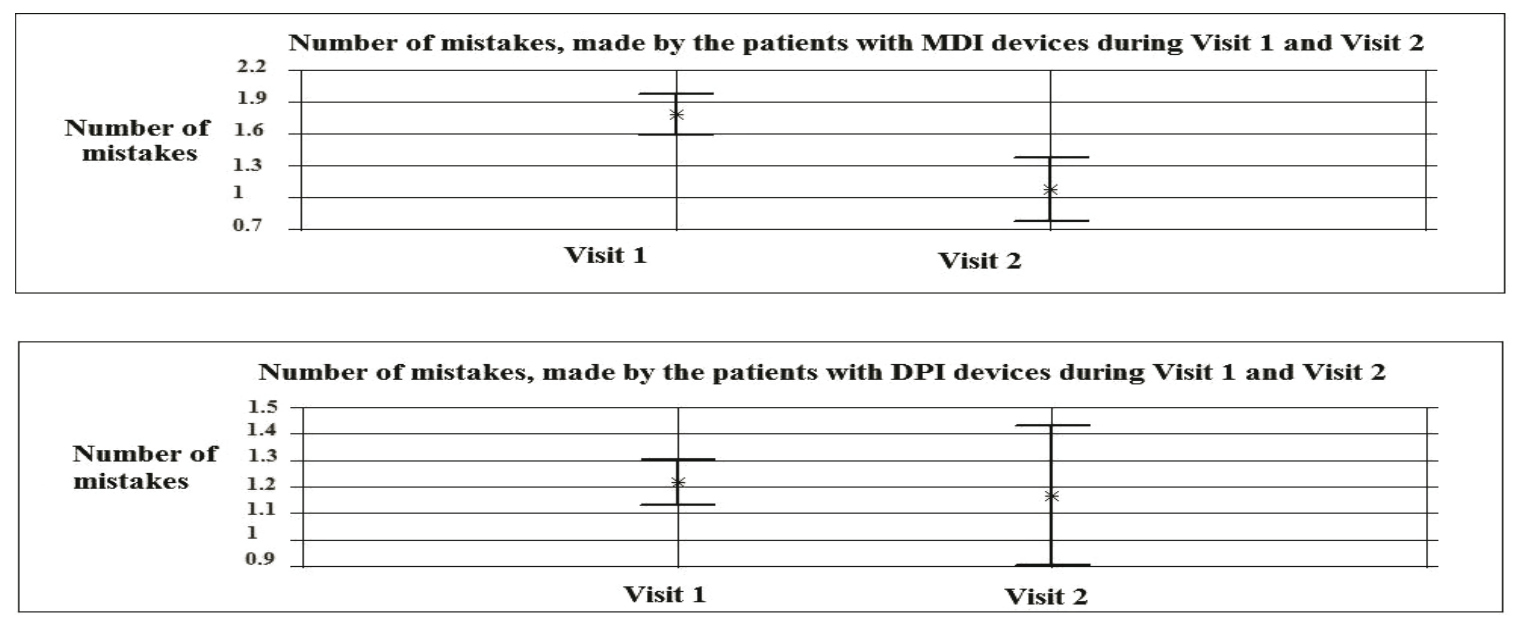

Figure 3. The number of mistakes made by the patients during inhalation in MDI and DPI groups during visit 1 and visit 2 . Patients in the MDI group made significantly more mistakes during visit 1 , while there was no difference in the number of mistakes in the DPI group between the two visits

We found that the average score [SD] for ACT was 13.25 points [4.82] for the MDI group, and 12.42 points [3.28] for the DPI group. There was no significant difference between the scores for the two groups $(\mathrm{F}=0.56 ; \mathrm{p}=0.444)$. The results for CAT were similar - with no significant difference between the groups - 18.66 points [7.16] for MDI and 21 points [5.93] in DPI group $(\mathrm{F}=1.41$; $\mathrm{p}=0.24)$. There was no significant difference in the ACT score [SD] between patient with correct technique (12.62 points [4]) and incorrect technique (12.86 points [3.9]; $\mathrm{KW}=0.216$; $\mathrm{p}=0.642$ ). As regards the CAT score [SD], no significant correlation was found between the patients with good technique (20.36 points [6.5]) and bad technique (20.83 points [5.9]; $\mathrm{KW}=0.033 ; \mathrm{p}=0.856$ ).

No difference was found in the number of hospitalizations between the patients with correct and incorrect inhalation technique for the previous year $(\mathrm{KW}=0.458$; $\mathrm{p}=0.499)$ : for both groups, the average rate was 1 hospitalization per year.

There was no difference in the duration of using inhalers among the patients with correct and incorrect inhalation skills. For the patients with correct inhalation technique the mean duration was 9 years $(\mathrm{ME}=9$ years, range 0 41 years) and for those with incorrect technique the result was similar (ME $=9$ years, range $0-45$ years; $\mathrm{KW}=0.126$; $\mathrm{p}=0.723$ ).

\section{Discussion}

To our knowledge, this is the first study in Bulgaria in which the inhalation technique of 
patients with obstructive pulmonary diseases has been tested via an objective method (Vitalograph AIM device). Via Vitalograph AIM, inhalation technique can be easily tested, since it provides important and detailed information about the strength and duration of the airflow, coordination between activating the canister and inhalation, and how long the patient holds his/her breath after inhalation. The method is safe for the patients because a placebo canister is used for MDI devices. For maximum hygiene, disposable inhaler stimulators are available.

In this study, it was found that most of the patients did not use their inhaler devices properly, even though they claimed that they knew how to inhale. In the MDI group, only 2 patients (5.88\%) demonstrated correct inhalation technique during their first attempt, while in the DPI group 31 patients (36.05\%) demonstrated it. Another study revealed that between $60 \%$ and $80 \%$ of the patients with MDI devices had incorrect inhalation technique [4]. Chrystyn et al. found errors during inhalation in $92 \%$ of the patients with MDI. Inhalation mistakes in patients with DPI devices vary from $39 \%$ (for diskus devices) to $76 \%$ (for turbohaler users) [12].

The patients with MDI devices were significantly younger (57.5 years) and with predominance of cases with bronchial asthma, as compared to the patients with DPI devices.

The most frequent mistakes during inhalation in the MDI group were short duration of inhalation (55.88\%) and poor coordination between the canister activation and inhalation (29.41\%). This type of device is activated, when the patient presses the canister and inhalation must start at the same time. The patient should provide smooth and long inhalation airflow. It is an interesting finding that patients have difficulties when using MDI inhalers but most of them do not have a spacer (82.3\%) or do not use it frequently if they do. In other studies, the percentage of people with spacers varies between $6-14 \%[4]$.

The most common mistake in the DPI group we studied was that inhalation was not forceful enough (48.84\%). One possible explanation for this is the fact that this type of device is activated by the patient's inspiratory airflow, which should be forceful enough. Patients with decreased lung function have more difficulties in achieving the airflow optimal for inhalation.

At Visit 1, patients with MDI made significantly more mistakes (average 1 patient made 2 mistakes) during inhalation than patients with DPI device (average 1 patient made 1 mistake). When we compared the number of mistakes during visit 1 and visit 2 inside the groups, it was found that in the MDI group there was a significant reduction in the number of mistakes (visit 1 - average 2 mistakes per person; visit 2 - 1 mistake per person). In the DPI group, no such correlation was found (visit 1 - average 1 mistakes per person; visit $2-1$ mistake per person), but it is worth mentioning that during visit 2 , none of the patients made more than 1 mistake after the extra training.

The patients with MDI devices seemed to be "riskier" for inhalation misuse. It should be taken in consideration the fact that, in Bulgaria, the reliever inhalers are in MDI devices. Health care providers should provide continuous education, frequent inhalation technique testing and timely correction of mistakes because patients could run into a difficult situation, being unable to manage an acute exacerbation just because they do not use their devices properly.

We observed very low attendance to visit 2 . Appointments were made for 63 patients but only 25 of them turned up. Most of the patients pointed out some of the following reasons for their reluctance to perform visit 2: lack of time, impossibility to leave their workplace, problems with public transport, and financial difficulties. Press et al. reported much higher attendance at visit 2: $84-91 \%$ in their study [13].

Severe exacerbations of the obstructive disease are an indication for hospitalization. No difference was found in the number of hospitalizations between patients with correct and incorrect inhalation technique for the previous year - for both groups, it was 1 hospitalization a year, on the average. One possible explanation for this could be the lack of information, regarding the rate of mild and moderate exacerbations, which were treated and controlled on an outpatient basis, or patients managed themselves at home with reliever inhalers. If the patients with the corrected technique had been followed prospectively, a reduction of the hospitalizations probably could have been found.

No significant correlation between the duration of using inhalers and good inhalation skills was found in our study: for both groups the mean age was 9 years. However, some authors have found that longer duration of inhaler use is a factor, favouring the good inhalation skills [14].

A significant correlation between obstructive disease control (assessed with ACT or CAT) of 
the patients with correct and incorrect technique was not found. In the available literature, there are studies reporting that patients with poor inhalation technique presented with worse disease control $[4,6]$. The questionnaires in our study were filled upon discharge from hospital, when the patients can be expected to be in their optimal health condition and present with higher $\mathrm{ACT} / \mathrm{CAT}$ scores despite their incorrect inhalation technique.

There are certain strengths of this study. In Bulgaria, this was the first time that the inhalation technique of patients was tested via an objective method. One direct benefit for the patients was to be tested and educated to inhale properly. The study revealed that most of the patients misused their inhalers. Furthermore, it was found that few patients with MDI inhalers had spacers even though they needed such devices to support their inhalation maneuvers.

There are also some limitations of the study. There was insufficient information on the mild and moderate rate of exacerbations, treated on an outpatient basis. To assess the long-term effect of testing and education, the patients should be followed up prospectively for longer periods, not only for one month after visit 1 .

\section{Conclusions}

The efforts to educate and correct the poor inhalation technique are worthwhile because they improve the skills to use of inhaler devices, thus leading to reduction of the number of mistakes during inhalation. Long-term positive effects of the good inhalation technique are expected such as reduction of exacerbations, hospitalizations and emergency visits, reduction of costs and probably better quality of life for the patients.

\section{Acknowledgements}

The authors would like to express their sincere gratitude to Medical University Pleven, for the technical and financial support for this study.

\section{References}

1. Sanders M. Inhalation therapy: an historical review. Prim Care Respir J. 2007;16(2):71-81.

2. Lavorini F, Usmani O. Correct inhalation technique is critical in achieving good asthma control. Prim Care Respir J. 2013;22(4):383-92.

3. Georgieva M, Kostov K. [Inhaler devices and inhalation techniques]. InSpiro. 2015;5(33):32-
41. Bulgarian.

4. Levy ML, Hardwell A, McKnight E, Holmes J. Asthma patients' inability to use a pressurised metered-dose inhaler (pMDI) correctly correlates with poor asthma control as defined by the Global Initiative for Asthma (GINA) strategy: a retrospective analysis. Prim Care Respir J. 2013;22(4):406-11.

5. Goodman DE, Israel E, Rosenberg M, Johnston R, Weiss ST, Drazen JM. The influence of age, diagnosis, and gender on proper use of metereddose inhalers. Am J Respir Crit Care Med. 1994;150(5 Pt 1):1256-61. DOI: 10.1164/ ajrccm.150.5.7952549 PubMed PMID: 7952549.

6. Melani A, Bonavia M, Cilenti V, Cinti C, Lodi M, Martucci $\mathrm{P}$, et al. Inhaler mishandling remains common in real life and is associated with reduced disease control. Respir Med. 2011;105(6):930-8.

7. Perenovska P. [The role of inhaler corticosteroids in children's asthma control]. MedInfo. 2010;(8):28-30. Bulgarian.

8. Global Initiative for Asthma. Global strategy for asthma management and prevention, 2016 [Internet]. Global Initiative for Asthma; c 2016 [cited 2016 Jun 21]. Available from: www.ginasthma.org.

9. Reddel H, Taylor D, Bateman E, Boulet LP, Boushey H, Busse W, et al. An official American Thoracic Society/European Respiratory Society statement: asthma control and exacerbations standardizing endpoints for clinical asthma trials and clinical practice. Am J Respir Crit Care Med. 2009;180:59-99. DOI: 10.1164/rccm.200801060ST.

10. thoracic.org [Internet]. New York: American Thoracic Society; c1998-2016 [updated 2014 Apr 29; cited 2016 Jun 21]. COPD Assessment Test (CAT); [about 2 screens]. Available from: http://www.thoracic.org/assemblies/srn/question aires/copd.php.

11. Yankov K. [Treatment of stable COPD]. Nauka Pulmologiya. 2007;(1):19-22. Bulgarian.

12. Chrystyn H, Price D, Molimard M, Haughney J, Bosnic-Anticevich S, Lavorini F, et al. Comparison of serious inhaler technique errors made by device-naïve patients using three different dry powder inhalers: a randomised, crossover, open-label study. BMC Pulm Med. 2016;16:12. DOI: 10.1186/s12890-016-0169-5. PMCID: PMC4712500.

13. Press V, Arora V, Trela K, Adhikari R, Zadravecz F, Liao C, et al. Effectiveness of interventions to teach metered-dose and Diskus inhaler techniques: A randomized trial. Ann Am Thorac Soc. 2016;13(6):816-24.

14. Camilleri K, Balzan M, Bardon M, Schembri E, Sullivan M, Mifsud S et al. Predictors of good inhaler technique in asthma and COPD. Eur R e s p i r J . $2015 ; 46$ ( 59 ). D O I : 10.1183/13993003.congress-2015.PA3928. 